\title{
Comparação dos métodos de parcelas e pontos-quadrantes para descrever uma comunidade lenhosa de Cerrado Típico
}

\author{
Firmino Cardoso Pereira ${ }^{1 *}$ \\ Eddie Lenza ${ }^{2}$ \\ Leonardo Maracahipes-Santos ${ }^{1}$ \\ Henrique Augusto Mews ${ }^{1}$ \\ Letícia Gomes ${ }^{3}$ \\ Stefany Lorrayny Lima ${ }^{1}$ \\ Kelyane Silva Melo Santos ${ }^{1}$

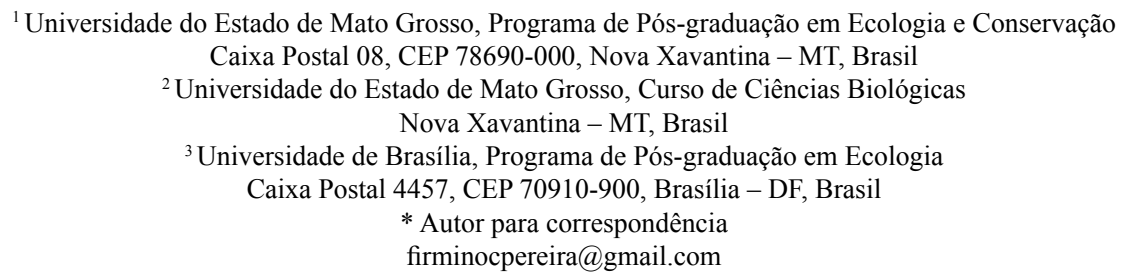

Submetido em 09/09/2014

Aceito para publicação em 19/02/2015

\section{Resumo}

Este artigo avalia a eficiência dos métodos de parcelas de área fixa (PA) e pontos quadrantes (QD) na descrição de uma comunidade lenhosa de Cerrado Típico. Utilizamos 10 PA e 140 QD, distribuídos em cinco transeções. Comparamos a densidade de indivíduos, a composição florística, a riqueza de famílias, gêneros e espécies e a estrutura vertical e horizontal da vegetação. O método de PA foi mais eficiente para amostrar densidade de indivíduos. O método de QD foi mais eficiente na caracterização da riqueza de espécies, da estrutura vertical da vegetação e do registro de espécies com baixa abundância. A composição de famílias, gêneros e espécies, bem como as espécies com maior índice de valor de importância na comunidade foram determinadas de maneira semelhante pelos dois métodos. Os métodos comparados são complementares. Sugerimos que o uso de PA, de QD ou de ambos os métodos pode ser direcionado ao parâmetro vegetacional investigado.

Palavras-chave: Descrição da vegetação; Eficiência amostral; Fitossociologia; Savana

\section{Abstract}

Comparing the methods plot and point-centered quarter to describe a woody community from typical Cerrado. This article evaluates the effectiveness of the methods fixed area plots (AP) and point-centered quarters (PQ) to describe a woody community from typical Cerrado. We used 10 APs and 140 PQs, distributed into 5 transects. We compared the density of individuals, floristic composition, richness of families, genera, 
and species, and vertical and horizontal vegetation structure. The AP method was more effective to sample the density of individuals. The PQ method was more effective for characterizing species richness, vertical vegetation structure, and record of species with low abundance. The composition of families, genera, and species, as well as the species with higher importance value index in the community were similarly determined by the 2 methods. The methods compared are complementary. We suggest that the use of AP, PQ, or both methods may be aimed at the vegetation parameter under study.

Key words: Phytosociology; Sampling effectiveness; Savanna; Vegetation description

\section{Introdução}

O Bioma Cerrado possui alta riqueza de espécies vegetais (MENDONÇA et al., 2008) e elevada heterogeneidade florística e fitofisionômica em escala espacial, local (OLIVEIRA-FILHO; RATTER, 2002; RIBEIRO; WALTER, 2008) e regional (RATTER et al., 2003; BRIDGEWATER et al., 2004; SILVA et al., 2006). O complexo mosaico florístico e fitofisionômico do Cerrado é influenciado por distintos fatores ambientais e antrópicos, os quais afetam a riqueza local de espécies, a composição florística e a estrutura da vegetação (MOREIRA, 2000; OLIVEIRA-FILHO; RATTER, 2002; DURIGAN et al., 2002a). Assim, devido à grande área de distribuição original do Cerrado (cerca de $2.000 .000 \mathrm{de} \mathrm{km}^{2}$ ), às variações florísticas nas diferentes escalas espaciais e à acelerada destruição que o bioma vem experimentando nas últimas décadas (SILVA et al., 2006), é necessário conduzir estudos de campo que sejam rápidos e eficientes na descrição da composição e riqueza de espécies e da estrutura das comunidades de plantas nas áreas remanescentes.

Nas últimas três décadas, inúmeros estudos descritivos da vegetação em escala local foram desenvolvidos nas diferentes fitofisionomias do Cerrado. Em geral, esses estudos adotaram o método de parcelas de área fixa proposto inicialmente por Felfili e SilvaJúnior (1988) e revisado e atualizado por Felfili et al. (2005). O emprego de parcelas de área fixa é muito útil, pois além de permitir comparações entre comunidades (FELFILI; FELFILI, 2001; FONSECA; SILVAJÚNIOR, 2004; MARIMON-JUNIOR; HARIDASAN, 2005; GOMES et al., 2011; MORO; MARTINS, 2011; PINHEIRO; DURIGAN, 2012; SANTOS et al., 2012; MEWS et al., 2014), possibilita o acompanhamento da dinâmica temporal da vegetação (FELFILI et al., 2000; LIBANO; FELFILI, 2006; AQUINO et al., 2007;
MORO; MARTINS, 2011; GOMES et al., 2014) e a realização de análises fitogeográficas (RATTER et al., 2003; BRIDGEWATER et al., 2004; SILVA et al., 2006). Essas análises temporais e espaciais têm fornecido informações que subsidiam o estabelecimento de estratégias de conservação da biodiversidade do Cerrado (FELFILI; SILVA-JÚNIOR, 2001; RATTER et al., 2003; BRIDGEWATER et al., 2004 FELFILI et al., 2007; 2008; MEWS et al., 2014). No entanto, a condução de estudos de comunidades vegetais é muitas vezes condicionada pela disponibilidade de recursos financeiros e de uma equipe de trabalho com disponibilidade de realizar os levantamentos. O tempo necessário para as pesquisas em campo é fator considerável, principalmente devido às dificuldades logísticas (SILVA-JÚNIOR, 2004; WALTER; GUARINO, 2006). Com isso, métodos mais rápidos e menos onerosos de amostragem da vegetação podem ser úteis, desde que permitam coleta confiável dos dados (WALTER; GUARINO, 2006).

Um método alternativo ao de parcelas é o de quadrantes, que, por ser mais rápido e não necessitar de demarcação de área amostral, permite amostragem espacial mais ampla e em tempo mais curto (COTTAM; CURTIS, 1956; MORO; MARTINS, 2011). No entanto, o método de parcelas, por ser de área fixa, permite análises quantitativas mais diretas (p.ex., produção em área basal, volume, biomassa, carbono ou dinâmica da vegetação) (FELFILI et al., 2011). Apesar do considerável número de investigações que empregaram os métodos de quadrantes (RATTER; GIFFORD, 1988; SILVA et al., 2002; COSTA et al., 2010) e de parcelas de área fixa no Cerrado, temos conhecimento de apenas um único estudo que avaliou a eficiência e as vantagens e desvantagens desses dois métodos na descrição das propriedades de uma comunidade vegetal florestal (Mata de Galeria) do Cerrado (BUENO et al., 2011). 
Assim, não há na literatura estudos comparativos entre os resultados provenientes dos métodos de parcelas e de quadrantes em comunidades savânicas do Cerrado. Nesse contexto, nós comparamos a eficiência dos métodos de parcelas (área fixa) e de quadrantes (área variável) para a determinação da riqueza e da composição de espécies e para a descrição da estrutura horizontal e vertical de uma comunidade lenhosa de Cerrado Típico.

\section{Material e Métodos}

Realizamos o estudo em uma área de Cerrado Típico (sensu RIBEIRO; WALTER, 2008) no Parque Municipal do Bacaba (1442’31,35'S e 52²1'1,48”O), município de Nova Xavantina, região leste de Mato Grosso. O Parque é uma Unidade de Conservação que abrange 492 ha, com predominância de Cerrado sentido restrito em contato com fragmentos naturais de Cerradão e Mata de Galeria (MARIMON-JUNIOR; HARIDASAN, 2005). Veja localização do Parque em relação ao estado de Mato Grosso em Brasil et al. (2013).
Amostramos a vegetação mediante os métodos de parcelas de área fixa (PA) (PHILIP, 1994) e de quadrantes (QD) (COTTAM; CURTIS, 1956). Ambos os métodos estão descritos em detalhes em Moro e Martins (2011). Amostramos 10 parcelas de 20×50 m distantes 50 m entre si (Figura 1; veja também GOMES et al., 2011). O critério utilizado para escolha das dimensões das parcelas seguiu Felfili et al. (2005). Para a instalação das transeções onde os pontos-quadrantes foram amostrados, demarcamos cinco transeções de caminhada entre as parcelas. Quatro transeções tinham $300 \mathrm{~m}$ de comprimento e uma delas tinha $200 \mathrm{~m}$ devido às mudanças fitofisionômicas na paisagem, as quais limitavam nossa capacidade de amostrar a vegetação de Cerrado Típico (Figura 1). Dividimos cada transeção em subseções de 100 m, o que resultou em 14 subseções que nós utilizamos como unidades amostrais. Ao longo das transeções, marcamos quadrantes com distância regular de 10 metros entre si, o que totalizou 140 pontos e 560 indivíduos amostrados.

FIGURA 1: Desenho amostral do Cerrado Típico inventariado pelos métodos de parcelas de área fixa (P) e de pontos-quadrantes no Parque do Bacaba, Nova Xavantina-MT, Brasil. Os pontos quadrantes estão distribuídos nas 14 subseções representadas pela letra "S".

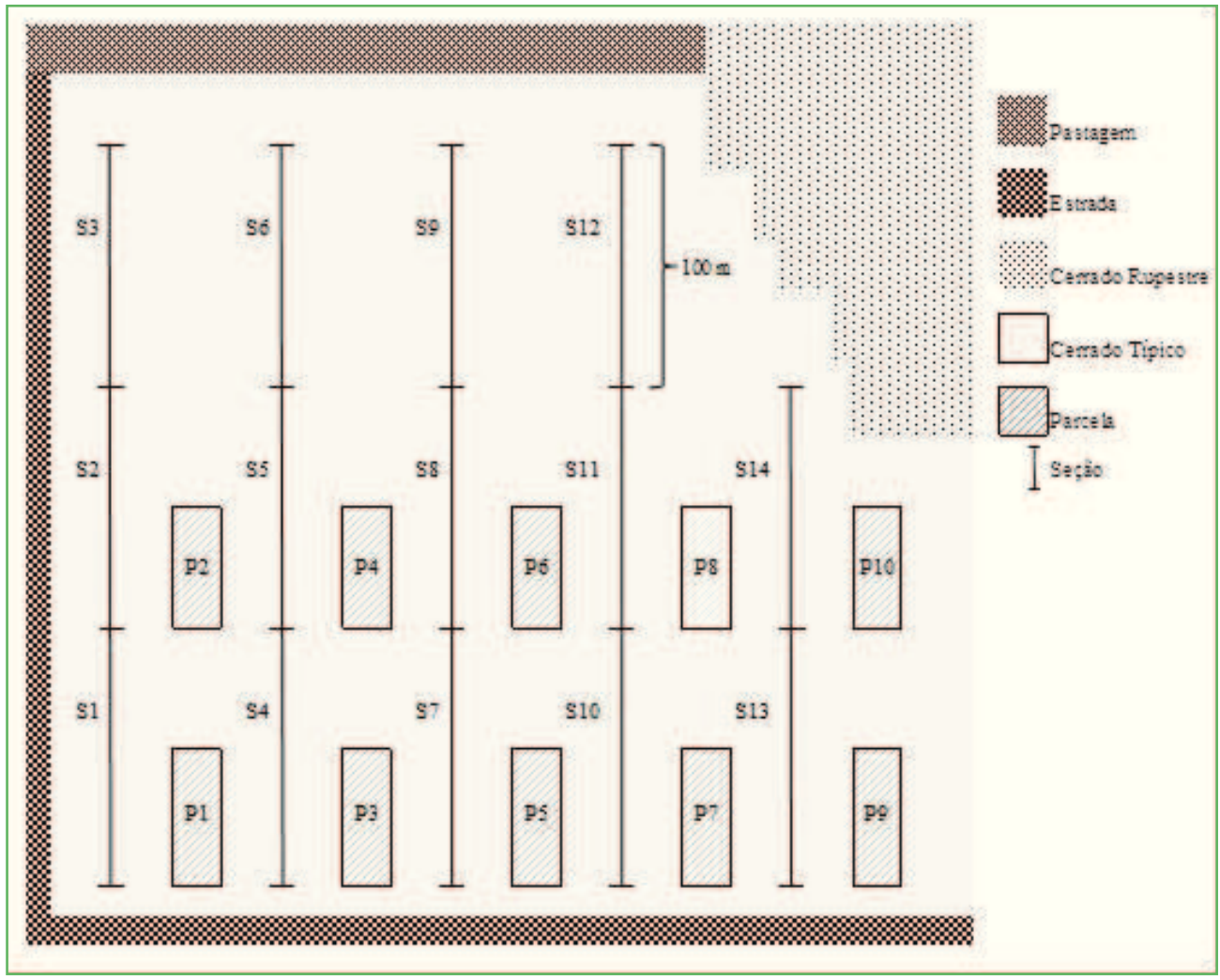


Usando ambos os métodos nós amostramos todos os indivíduos lenhosos vivos - incluindo lianas e monocotiledôneas arborescentes (Arecaceae e Velloziaceae $)$ - cujo diâmetro a $30 \mathrm{~cm}$ do solo $\left(\mathrm{Db}_{30 \mathrm{~cm}}\right)$ foi igual ou superior a $3 \mathrm{~cm}$. Identificamos as espécies e mensuramos o $\mathrm{Db}_{30}$ e a altura total de todos os indivíduos que atingiram o critério de inclusão adotado $\left(\mathrm{Db}_{30} \geq 3\right.$ $\mathrm{cm}$ de diâmetro). Em casos de indivíduos ramificados, incluímos o indivíduo quando pelo menos um dos ramos possuiu o $\mathrm{Db}_{30}$ mínimo de inclusão. Nós calculamos a área basal mediante a soma dos diâmetros quadráticos para evitar superestimativa da área basal dos indivíduos (MORO; MARTINS, 2011).

Identificamos as espécies em campo e, quando isso não foi possível, consultamos bibliografia especializada e comparamos as coletas com o acervo do Herbário NX (Universidade do Estado de Mato Grosso, Campus de Nova Xavantina). Para as famílias botânicas, adotamos o sistema de classificação APG III (2009). Conferimos os nomes dos táxons na Lista de Espécies da Flora do Brasil (2014).

Tendo obtido os valores das variáveis fitossociológicas por cada um dos métodos (parcela e pontos-quadrantes), comparamos a densidade de indivíduos obtida para a comunidade pelos dois métodos (extrapolada para 1 ha para o método de QD, a partir da área média dos indivíduos por ponto (MORO; MARTINS, 2011)). As comparações de densidade entre os métodos foram executadas mediante o uso do teste de Mann-Whitney. Para este teste, utilizamos as 10 parcelas (PA) e as 14 subseções (QD) como unidades amostrais, cujas densidades foram extrapoladas para 1 ha. Também empregamos teste de Mann-Whitney para comparar as medidas de diâmetro e de altura das plantas entre os dois métodos e para verificar se os métodos de PA e QD incluem indivíduos de tamanhos significativamente diferentes na amostragem. Para essas análises, empregamos o programa PAST 3.0 (HAMMER, 2013) e adotamos o nível de significância de $5 \%$.

Calculamos os parâmetros fitossociológicos de densidade, frequência e dominância relativas, os quais somados compõem o Índice de Valor de Importância (IVI) das espécies (MÜELLER-DOMBOIS; ELLENBERG,
1974; MORO; MARTINS, 2011). Os valores de IVI foram calculados usando os dados obtidos pelos dois métodos. Usamos, para tanto, o programa FITOPAC 2.1 (SHEPHERD, 2010). Comparamos a riqueza observada com a estimada para avaliar a suficiência amostral florística dos métodos de PA e QD. Usamos, para isso, o estimador Bootstrap porque ele apresentou o maior valor de correlação de Spearman entre os valores estimados e observados (WALTHER; MOORE, 2005). Comparamos a riqueza estimada de espécies da comunidade entre os dois métodos por meio de rarefação (Mao tau) com esforço amostral padronizado em termos de número de indivíduos (GOTELLI; COLWELL, 2001). Comparamos também as curvas de rarefação graficamente e com uso de intervalos de confiança de 95\% (ZAR, 1999; MAGURRAN, 2011). Por fim, avaliamos a similaridade florística da comunidade amostrada pelos dois métodos mediante os índices de Sørensen (CCs, qualitativo) e de Morisita (Im, quantitativo) (MAGURRAN, 1988). Realizamos estas análises em ambiente R 3.0.2 (R CORE TEAM, 2013) com o pacote vegan (OKSANEN et al., 2013).

\section{Resultados}

Os métodos de parcelas de área fixa (PA) e de quadrantes (QD) resultaram na amostragem de 1.523 (abundância média de $152,3 \pm 39,5-\mathrm{n}=10$ ) e 3.279 (abundância média de $40 \pm 0, \mathrm{n}=14$ ) indivíduos.ha ${ }^{-1}$, respectivamente. O método QD incluiu exatamente 560 indivíduos quando o valor não foi estimado para área de um hectare (Tabela 1). O método de QD resultou em densidade de indivíduos maior do que a encontrada pelo método de PA $(U=2,0 ; p<0,001)$ para a mesma comunidade. $\mathrm{O}$ método de $\mathrm{PA}$ incluiu na amostragem plantas mais altas $(U=392.123,5 ; p=0,004)$ e de maior diâmetro $(U=288.888,0 ; p<0,001)$ que o método de QD (Tabela 1).

Entre as 10 espécies com maior índice de valor de importância (IVI), sete foram comuns aos dois métodos: Aspidosperma tomentosum, Byrsonima pachyphylla, Davilla elliptica, Erythroxylum suberosum, Myrcia lanuginosa, Pouteria ramiflora e Qualea parviflora. Três espécies estiveram entre as 10 mais importantes em 
TABELA 1: Parâmetros estruturais e florísticos do Cerrado Típico amostrado pelo método de parcelas de área fixa (PA) e quadrantes (QD) no Parque do Bacaba, Nova Xavantina-MT, Brasil.

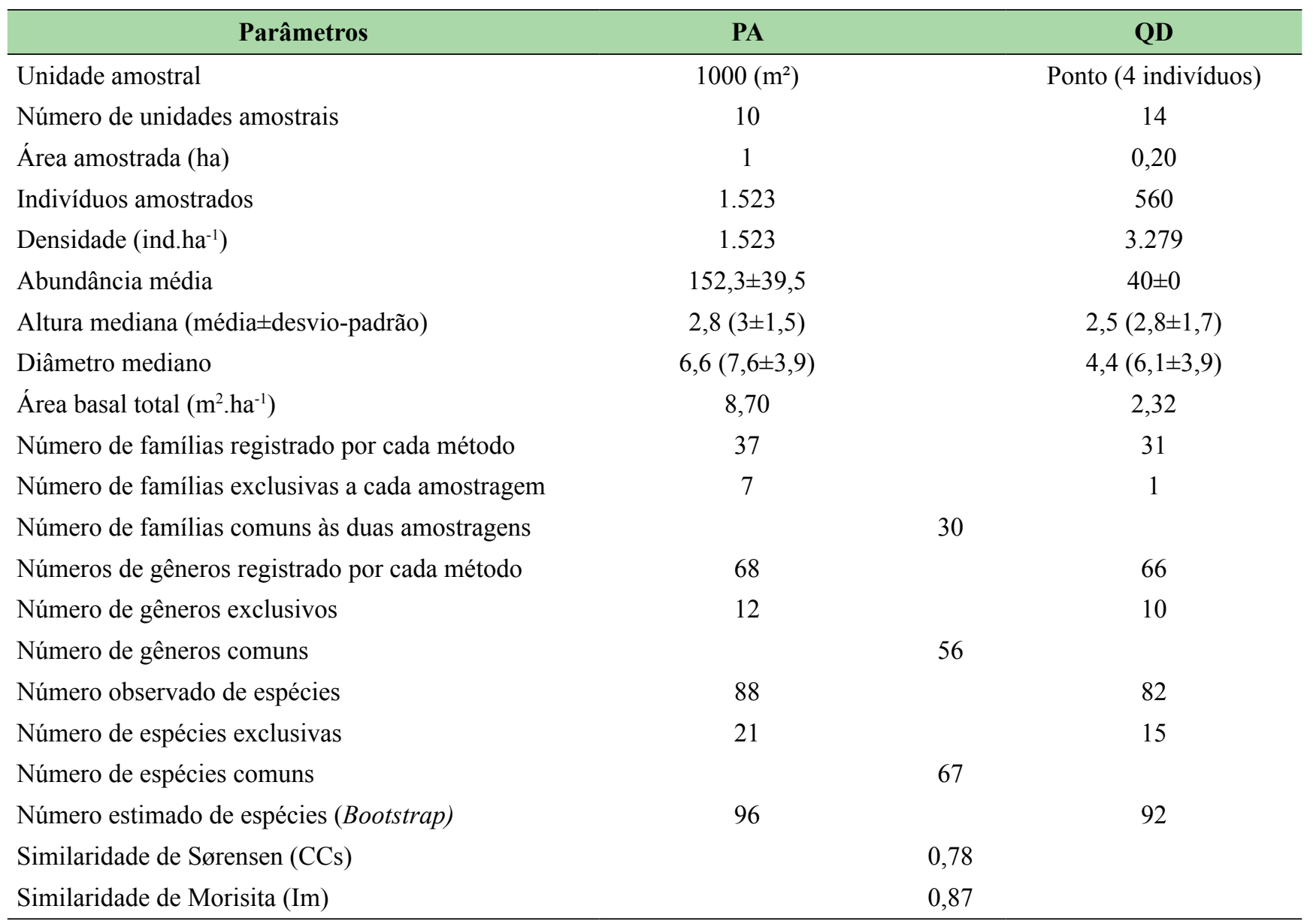

apenas um dos dois métodos (Anacardium occidentale, Curatella americana e Salvertia convallariodora para o método de PA e Ouratea spectabilis, Qualea multiflora e Syagrus flexuosa para o método de QD) (Tabela 2). De forma semelhante, das 10 espécies com maior abundância, sete foram comuns a ambos os métodos ( $A$. tomentosum, B. pachyphylla, D. elliptica, E. suberosum, M. lanuginosa, Q. parviflora e $S$. flexuosa), três apenas no método de PA (Byrsonima coccolobifolia, Kielmeyera rubriflora e $S$. convallariodora) e três apenas no método de QD (Heteropterys byrsonimifolia, O. spectabilis e $Q$. multiflora) (Tabela 2).
Encontramos 38 famílias, das quais 37 foram amostradas pelo método de PA e 31 pelo método QD. Trinta famílias (79\% do total) foram comuns aos dois métodos, enquanto sete (18\%) foram amostradas apenas pelo método PA (Asteraceae, Caryocaraceae, Lauraceae, Olacaceae, Opiliaceae, Salicaceae e Velloziaceae) e uma (3\%) apenas no QD (Styracaceae). As famílias mais ricas em espécies foram semelhantes para ambos os métodos de amostragem. 
TABELA 2: Parâmetros fitossociológicos do Cerrado Típico investigado pelos métodos de parcelas de área fixa (PA) e quadrantes (QD) no Parque do Bacaba, Nova Xavantina-MT, Brasil. Espécies em ordem decrescente do Índice de Valor de Importância (IVI) do método PA. N = número de indivíduos amostrados por cada método (abundância). Os dados de campo e as tabelas fitossociológicas completas para os dois métodos estão disponíveis como documento suplementar em: http://dx.doi.org/10.6084/m9.figshare.1254531

\begin{tabular}{|c|c|c|c|c|c|c|}
\hline & \multirow{2}{*}{ Espécies } & \multirow{2}{*}{ Família } & \multicolumn{3}{|c|}{$\mathbf{N}$} & \multirow{2}{*}{$\frac{\text { IVI }}{\text { QD }}$} \\
\hline & & & PA & QD & PA & \\
\hline 1. & Qualea parviflora Mart. (*) & Vochysiaceae & 220 & 57 & 33,20 & 31,56 \\
\hline 2. & Davilla elliptica A.St.-Hil. (*) & Dilleniaceae & 127 & 26 & 16,92 & 13,19 \\
\hline 3. & Anacardium occidentale L. & Anacardiaceae & 37 & 5 & 14,07 & 3,92 \\
\hline 4. & Myrcia lanuginosa O.Berg $(*)$ & Myrtaceae & 94 & 67 & 10,93 & 27,26 \\
\hline 5. & Byrsonima pachyphylla A.Juss. (*) & Malpighiaceae & 81 & 19 & 10,77 & 10,62 \\
\hline 6. & Salvertia convallariodora A.St.-Hil. & Vochysiaceae & 41 & 10 & 9,50 & 7,73 \\
\hline 7. & Erythroxylum suberosum A.St.-Hil. (*) & Erythroxylaceae & 71 & 30 & 9,11 & 11,87 \\
\hline 8. & Curatella americana $\mathrm{L}$. & Dilleniaceae & 35 & 8 & 8,97 & 6,09 \\
\hline 9. & Aspidosperma tomentosum Mart. (*) & Apocynaceae & 61 & 16 & 8,90 & 8,87 \\
\hline 10. & Pouteria ramiflora (Mart.) Radlk. (*) & Sapotaceae & 23 & 5 & 8,79 & 9,11 \\
\hline 11. & Syagrus flexuosa (Mart.) Becc. (*) & Arecaceae & 69 & 16 & 8,18 & 9,33 \\
\hline 12. & Byrsonima coccolobifolia Kunth & Malpighiaceae & 42 & 4 & 7,57 & 2,13 \\
\hline 13. & Kielmeyera rubriflora Cambess. & Calophyllaceae & 45 & 12 & 7,40 & 6,34 \\
\hline 14. & Ouratea hexasperma (A.St.-Hil.) Baill. & Ochnaceae & 40 & 11 & 7,20 & 5,18 \\
\hline 15. & Pseudobombax longiflorum (Mart. \& Zucc.) A.Robyns & Malvaceae & 24 & 9 & 5,83 & 5,41 \\
\hline 16. & Eugenia aurata O.Berg & Myrtaceae & 30 & 2 & 5,78 & 1,32 \\
\hline 17. & Couepia grandiflora (Mart. \& Zucc.) Benth. & Chrysobalanaceae & 27 & 6 & 5,72 & 2,96 \\
\hline 18. & Qualea grandiflora Mart. & Vochysiaceae & 22 & 6 & 5,48 & 4,64 \\
\hline 19. & Qualea multiflora Mart. (*) & Vochysiaceae & 26 & 18 & 5,46 & 9,32 \\
\hline 20. & Dipteryx alata Vogel & Fabaceae & 15 & 6 & 5,35 & 5,56 \\
\hline 21. & Ouratea spectabilis (Mart.) Engl. (*) & Ochnaceae & 24 & 16 & 5,28 & 9,95 \\
\hline 22. & Tachigali aurea Tul. & Fabaceae & 22 & 6 & 4,46 & 2,81 \\
\hline 23. & Eriotheca gracilipes (K.Schum.) A.Robyns & Malvaceae & 17 & 8 & 4,18 & 5,59 \\
\hline 24. & Heteropterys byrsonimifolia A.Juss. & Malpighiaceae & 24 & 18 & 4,02 & 7,45 \\
\hline 25. & Vatairea macrocarpa (Benth.) Ducke & Fabaceae & 15 & 9 & 3,89 & 4,71 \\
\hline 26. & Annona coriacea Mart. & Annonaceae & 13 & 3 & 3,68 & 1,27 \\
\hline 27. & Astronium fraxinifolium Schott & Anacardiaceae & 12 & 6 & 3,67 & 4,58 \\
\hline 28. & Tabebuia aurea (Silva Manso) Benth. \& Hook.f. ex S.Moore & Bignoniaceae & 10 & 4 & 3,58 & 3,58 \\
\hline 29. & Lafoensia pacari A.St.-Hil. & Lythraceae & 13 & 13 & 3,51 & 5,73 \\
\hline 30. & Mouriri elliptica Mart. & Melastomataceae & 13 & 3 & 3,44 & 1,44 \\
\hline 31. & Licania humilis Cham. \& Schltdl. & Chrysobalanaceae & 14 & 5 & 2,84 & 2,74 \\
\hline 32. & Callisthene fasciculata Mart. & Vochysiaceae & 6 & 3 & 2,80 & 1,54 \\
\hline 33. & Bowdichia virgilioides Kunth & Fabaceae & 7 & 1 & 2,79 & 0,90 \\
\hline & Mimosa laticifera Rizzini \& A.Mattos & Fabaceae & 11 & 5 & 2,64 & 2,75 \\
\hline 35. & Hymenaea stigonocarpa Mart. ex Hayne & Fabaceae & 8 & 1 & 2,57 & 0,41 \\
\hline
\end{tabular}


36. Tocoyena formosa (Cham. \& Schltdl.) K.Schum.

37. Myrcia multiflora (Lam.) DC.

38. Magonia pubescens A.St.-Hil.

39. Luetzelburgia praecox (Harms) Harms

40. Erythroxylum tortuosum Mart.

41. Syagrus comosa (Mart.) Mart.

42. Buchenavia tomentosa Eichler

43. Kielmeyera coriacea Mart.

44. Myrcia camapuanensis N.Silveira

45. Plenckia populnea Reissek

46. Emmotum nitens (Benth.) Miers

47. Simarouba versicolor A.St.-Hil.

48. Plathymenia reticulata Benth.

49. Connarus suberosus Planch.

50. Strychnos pseudoquina A.St.-Hil.

51. Erythroxylum engleri O.E.Schulz

52. Roupala montana Aubl.

53. Diospyros hispida A.DC.

54. Terminalia argentea Mart.

55. Handroanthus ochraceus (Cham.) Mattos

56. Aspidosperma macrocarpon Mart.

57. Caryocar brasiliensis Cambess.

58. Leptolobium dasycarpum Vogel

59. Andira cujabensis Benth.

60. Guapira noxia (Netto) Lundell

61. Pseudobombax tomentosum (Mart. \& Zucc.) A.Robyns

62. Eugenia punicifolia (Kunth) DC.

63. Diplopterys pubipetala (A.Juss.) W.R.Anderson \& C.C.Davis

64. Eugenia gemmiflora O.Berg

65. Casearia sylvestris Sw.

66. Dalbergia miscolobium Benth.

67. Myrcia splendens (Sw.) DC.

68. Pterodon pubescens (Benth.) Benth.

69. Vellozia sp.

70. Vochysia rufa Mart.

71. Protium heptaphyllum (Aubl.) Marchand

72. Guapira graciliflora (Mart. ex Schmidt) Lundell

73. Cybistax antisyphilitica (Mart.) Mart.

74. Dimorphandra mollis Benth.

75. Psidium sp.

76. Stryphnodendron rotundifolium Mart.

\begin{tabular}{lcccc} 
Rubiaceae & 10 & 9 & 2,45 & 3,91 \\
Myrtaceae & 13 & - & 2,43 & - \\
Sapindaceae & 8 & 2 & 2,33 & 1,37 \\
Fabaceae & 7 & - & 2,09 & - \\
Erythroxylaceae & 12 & 1 & 2,07 & 0,41 \\
Arecaceae & 8 & 3 & 2,07 & 1,74 \\
Combretaceae & 6 & 2 & 1,99 & 3,22 \\
Calophyllaceae & 6 & 4 & 1,99 & 1,87 \\
Myrtaceae & 6 & 1 & 1,92 & 0,69 \\
Celastraceae & 7 & 2 & 1,67 & 0,88 \\
Icacinaceae & 2 & 3 & 1,44 & 1,42 \\
Simaroubaceae & 5 & 2 & 1,34 & 0,83 \\
Fabaceae & 6 & 2 & 1,31 & 0,85 \\
Connaraceae & 6 & 3 & 1,30 & 1,28 \\
Loganiaceae & 5 & - & 1,30 & - \\
Erythroxylaceae & 4 & 3 & 1,13 & 1,30 \\
Proteaceae & 5 & 9 & 1,12 & 3,93 \\
Ebenaceae & 2 & 8 & 1,00 & 3,50 \\
Combretaceae & 2 & 5 & 0,95 & 4,99 \\
Bignoniaceae & 2 & 1 & 0,93 & 0,46 \\
Apocynaceae & 2 & - & 0,88 & - \\
Caryocaraceae & 3 & - & 0,88 & - \\
Fabaceae & 4 & 2 & 0,86 & 0,82 \\
Fabaceae & 3 & 3 & 0,84 & 1,32 \\
Nyctaginaceae & 2 & - & 0,84 & - \\
Malvaceae & 3 & - & 0,83 & - \\
Myrtaceae & 2 & - & 0,75 & - \\
Malpighiaceae & 2 & 1 & 0,72 & 0,41 \\
Myrtaceae & 3 & - & 0,72 & - \\
Salicaceae & 2 & - & 0,71 & - \\
Fabaceae & 2 & - & 0,71 & - \\
Myrtaceae & 4 & - & 0,71 & - \\
Fabaceae & 1 & 1 & 0,71 & 0,47 \\
Velloziaceae & 4 & - & 0,71 & - \\
Vochysiaceae & 3 & 1 & 0,69 & 0,64 \\
Burseraceae & 1 & 10 & 0,53 & 3,42 \\
Nyctaginaceae & 3 & 10 & 0,51 & 3,92 \\
Mignoniaceae & 2 & 1 & 0,46 & 0,42 \\
Fabaceae & 2 & 2 & 0,46 & 1,11 \\
\hline & 1 & - & 0,40 & - \\
Myrtaceae & 1 & 0,40 & 0,43 \\
\hline
\end{tabular}




\begin{tabular}{|c|c|c|c|c|c|c|}
\hline 77. & Eugenia sp. & Myrtaceae & 1 & - & 0,39 & - \\
\hline 78. & Agonandra brasiliensis Miers ex Benth. \& Hook.f. & Opiliaceae & 1 & - & 0,37 & - \\
\hline 79. & Aspidosperma multiflorum A.DC. & Apocynaceae & 1 & 1 & 0,37 & 0,56 \\
\hline 80. & Heisteria ovata Benth. & Olacaceae & 1 & - & 0,37 & - \\
\hline 81. & Rourea induta Planch. & Connaraceae & 1 & 3 & 0,37 & 1,26 \\
\hline 82. & Salacia crassifolia (Mart. ex Schult.) G.Don & Celastraceae & 1 & 2 & 0,37 & 0,64 \\
\hline 83. & Erythroxylum daphnites Mart. & Erythroxylaceae & 1 & 2 & 0,36 & 1,02 \\
\hline 84. & Mezilaurus crassiramea (Meisn.) Taub. ex Mez & Lauraceae & 1 & - & 0,36 & - \\
\hline 85. & Peltogyne confertiflora (Mart. ex Hayne) Benth. & Fabaceae & 1 & - & 0,36 & - \\
\hline 86. & Eremanthus mattogrossensis Kuntze & Asteraceae & 1 & - & 0,35 & - \\
\hline 87. & Machaerium acutifolium Vogel & Fabaceae & 1 & 3 & 0,35 & 1,26 \\
\hline 88. & Salacia elliptica (Mart. ex Schult.) G.Don & Celastraceae & 1 & - & 0,35 & - \\
\hline 89. & Antonia ovata Pohl & Loganiaceae & - & 2 & - & 0,90 \\
\hline 90. & Aspidosperma subincanum Mart. & Apocynaceae & - & 1 & - & 0,50 \\
\hline 91. & Bauhinia brevipes Vogel & Fabaceae & - & 1 & - & 0,42 \\
\hline 92. & Erythroxylum sp. & Erythroxylaceae & - & 3 & - & 1,27 \\
\hline 93. & Euplassa inaequalis (Pohl) Engl. & Proteaceae & - & 2 & - & 1,87 \\
\hline 94. & Himatanthus obovatus (Müll.Arg.) Woodson & Apocynaceae & - & 1 & - & 0,45 \\
\hline 95. & Hirtella glandulosa Spreng. & Chrysobalanaceae & - & 1 & - & 0,44 \\
\hline 96. & Luehea sp. & Malvaceae & - & 2 & - & 1,06 \\
\hline 97. & Matayba guianensis Aubl. & Sapindaceae & - & 1 & - & 0,41 \\
\hline 98. & Myrcia tomentosa (Aubl.) DC. & Myrtaceae & - & 1 & - & 0,53 \\
\hline & Myrtaceae NI 2 & Myrtaceae & - & 1 & - & 0,44 \\
\hline 100. & Myrtaceae NI 1 & Myrtaceae & - & 1 & - & 0,76 \\
\hline 101. & Neea theifera Oerst. & Nyctaginaceae & - & 2 & - & 1,05 \\
\hline 102. & Peritassa campestris (Cambess.) A.C.Sm. & Celastraceae & - & 3 & - & 1,24 \\
\hline 103. & Styrax camporum Pohl & Styracaceae & - & 1 & - & 0,44 \\
\hline Total & geral & & 1523 & 560 & 300 & 300 \\
\hline
\end{tabular}

* Dez espécies com maior IVI no método QD.

Registramos total de 78 gêneros considerando os dois métodos de amostragem. Considerando cada método, o número de gêneros foi de 68 para PA e 66 para QD. Amostramos 12 gêneros (15\% do total) apenas no método PA, 10 apenas no QD (13\%) e 56 (72\%) comuns aos dois métodos. Amostramos 103 espécies com o emprego dos dois métodos. O número observado foi de 88 espécies nas PA e 82 nos QD. Do total de espécies, $21(20 \%)$ foram registradas apenas no PA, 15 (15\%) apenas no QD e 67 (65\%) nos dois métodos (Tabelas 1 e 2). A similaridade florística de Sørensen $(C C s=0,78)$ e de Morisita $(\operatorname{Im}=0,87)$ entre os dois métodos foi alta.
O número estimado de espécies (Bootstrap) foi de 96 espécies para o método PA e 92 para o método de QD. O número observado de espécies representou $92 \%$ e $89 \%$ do número estimado para PA e QD, respectivamente. $\mathrm{O}$ número estimado de espécies (Mao Tau) para o método de PA, com esforço amostral ajustado para 560 indivíduos, foi de $70 \pm 6$ espécies (intervalo de confiança de 95\%). Isso indicou que após padronização do esforço amostral para 560 indivíduos, o método de PA incluiu menos espécies que o método de QD ( $n=82)$ (Figura 2). 
FIGURA 2: Curvas de rarefação para a comunidade de Cerrado Típico amostrada pelos métodos de parcelas área fixa (PA) e quadrantes (QD) no Parque do Bacaba, Nova Xavantina-MT, Brasil. Faixas cinza representam intervalos de confiança de $95 \%$.

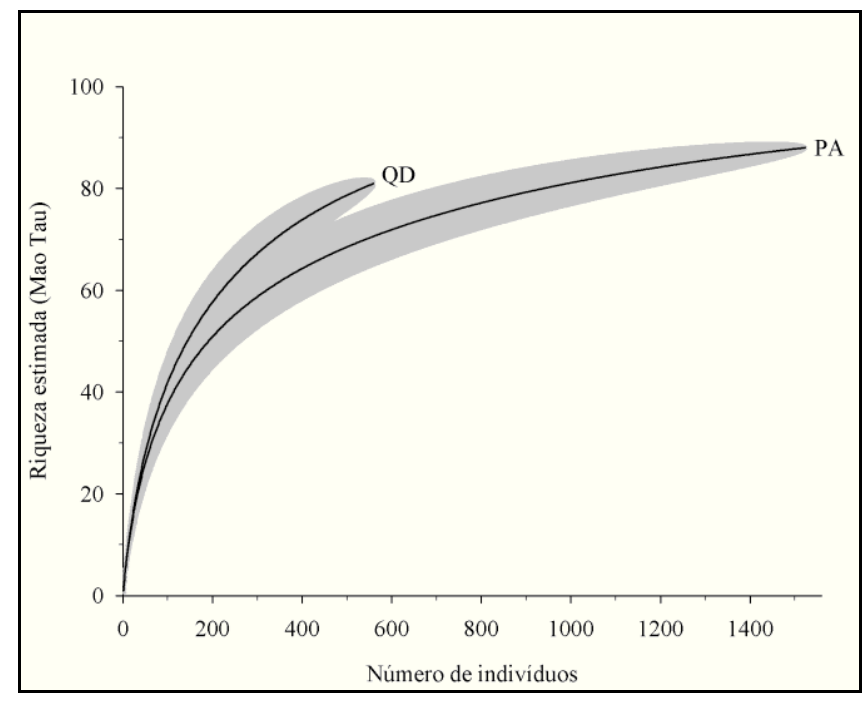

\section{Discussão}

Nossos resultados indicaram que ao extrapolar a densidade de indivíduos amostrada pelo método de QD para um hectare, houve superestimativa de $84 \%$ de indivíduos quando equiparada com a densidade de indivíduos amostrada pelo método de PA. Maior densidade determinada pelo método de QD foi também evidenciada por Gorenstein et al. (2007) e por Engeman et al. (1994) em simulações usando método de Monte Carlo e em situações nas quais a vegetação apresentava padrão regular de distribuição espacial das plantas. Não conhecemos o padrão de distribuição espacial dos indivíduos na comunidade de Cerrado estudada, mas notamos considerável coeficiente de variação $(26 \%)$ em relação à densidade média obtida nas 10 parcelas aqui amostradas, o que sugere distribuição agregada dos indivíduos. Assim, na comunidade savânica de Cerrado analisada, o método de QD superestimou a densidade, mesmo quando a distribuição dos indivíduos foi agregada.

As comparações dos valores médios de altura e diâmetro das plantas entre os métodos PA e QD também indicam divergências entre os dois métodos. As parcelas amostradas estavam equidistantes e ocuparam área restrita da comunidade. Por essa razão, elas podem ter sido estabelecidas em locais com indivíduos de maior porte. Desse modo, o método de QD parece ser mais adequado na descrição da estrutura vertical de comunidades de Cerrado, particularmente aquelas nas quais a estrutura vertical é heterogênea. Notamos no campo, ao aplicar o método de QD, grande variação no porte dos indivíduos entre pontos, o que confirma a heterogeneidade descrita acima. Essa limitação do método de PA é particularmente importante quando o interesse do estudo é descrever a estrutura vertical da vegetação. Uma alternativa seria optar pela amostragem inteiramente casualizada, como proposto por Felfili et al. (2011), ou estabelecer parcelas menores e mais espaçadas. Contudo, isso demandaria mais tempo e recursos financeiros.

Em nível taxonômico de família, gênero ou espécie, os dois métodos apresentaram resultados semelhantes. Contudo, é importante destacar que o método de QD poderia ter registrado maior riqueza, já que com número de indivíduos claramente menor que o método PA, incluiu semelhantes números de famílias, gêneros e espécies. Mesmo assim, acreditamos que as espécies amostradas exclusivamente por cada método possam ser reflexo de uma comunidade com elevado percentual de espécies raras, ou seja, pouco abundantes (aqui consideradas com $n \leq 2$ indivíduos). Walter e Guarino (2006), comparando método de levantamento rápido (caminhamento) com o método de parcelas em comunidade de Cerrado também notaram que a ausência de registros em um ou outro método se deu principalmente em função das espécies menos abundantes. Do modo como o desenho experimental foi estabelecido no presente estudo, não houve sobreposição das áreas amostradas entre os métodos de PA e de QD e espécies com baixas abundâncias locais podem não ter sido incluídas por um ou pelo outro método. Esse é o caso de Agonandra brasiliensis, Eremanthus mattogrossensis, Heisteria ovata, Mezilaurus crassiramea, Peltogyne confertiflora e Salacia elliptica, amostradas apenas no método de PA, e de Aspidosperma subincanum, Bauhinia brevipes, Himatanthus obovatus, Hirtella glandulosa, Matayba guianensis, Myrcia tomentosa e Styrax camporum, registradas apenas no método de QD. A raridade local de espécies lenhosas do Cerrado Típico 
é reforçada pelo fato de 28 espécies amostradas pelo método de PA ( $31 \%$ do total) e 34 espécies pelo método de QD (41\%) apresentarem abundâncias entre um e dois indivíduos. Na mesma área de estudo, Marimon-Junior e Haridasan (2005) empregaram o método de parcelas e registraram 18 espécies ( $23 \%$ do total) localmente raras.

Entre as 49 espécies identificadas em nível específico e localmente raras nos métodos de PA ou de QD, 16 (33\%) não ocorreram no estudo de MarimonJunior e Haridasan (2005). Por outro lado, das 17 espécies com baixas abundâncias $(n \leq 2)$ identificadas por Marimon-Junior e Haridasan (2005), nove também foram consideradas raras e quatro não foram registradas nos dois métodos empregados no presente estudo. Essas comparações sugerem que há um conjunto de espécies que são local e naturalmente raras, independentemente do método adotado (comparação entre os métodos de PA e de QD). No entanto, pode haver considerável distinção quanto ao conjunto de espécies raras, mesmo entre áreas próximas (comparação entre presente estudo e o de Marimon-Junior e Haridasan (2005)). Assim, o registro de espécies com baixa abundância local seria ampliado com o emprego do método de QD, o qual pode amostrar áreas maiores em um tempo relativamente menor do que o método de PA.

Quando igualamos o esforço amostral em termos de indivíduos ou quando estimamos a riqueza total da comunidade, o método de QD apresentou eficiência superior e igual, respectivamente, em relação ao método de PA. Nesse sentido, quando o objetivo é ampliar a riqueza florística e a composição de espécies, em qualquer um dos três níveis taxonômicos aqui analisados, recomendamos o método de QD em vez do método de PA, já que o método de QD é executado com maior facilidade. A praticidade, a flexibilidade e a eficiência do método de QD na caracterização florística (amostragem da riqueza e da composição de espécies) de comunidades de Cerrado também foi discutida por Felfili et al. (2011) e evidenciada em florestas estacionais semideciduais por Durigan et al. (2002b) e por Bueno et al. (2011). Mesmo assim, sugerimos cautela na descrição da composição florística de comunidades lenhosas de Cerrado, independentemente do método empregado, uma vez que foi registrado considerável proporção de táxons amostrados exclusivamente por um ou outro método, conforme discutido acima.

A eficiência semelhante entre o método de parcelas e de quadrantes na determinação das espécies mais importantes na comunidade de Cerrado Típico amostrada, seja em termos de índice de valor de importância ou de densidades, foi também evidenciada por Bueno et al. (2011) em Mata de Galeria. Usando método de parcelas, Marimon-Junior e Haridasan (2005) estudaram uma comunidade de Cerrado Típico, também no Parque do Bacaba. Entre as 10 espécies com maior IVI registradas pelos referidos autores, cinco apresentaram elevado valor de IVI pelo método de QD (Aspidosperma tomentosum, Byrsonima pachyphylla, Davilla elliptica, Qualea parviflora e Syagrus flexuosa) e quatro pelo método de PA (as mesmas pelo método de QD, exceto $S$. flexuosa) no presente estudo.

Concluímos que os dois métodos analisados são complementares, pois apresentam tanto vantagens quanto desvantagens, dependendo da propriedade comunitária analisada. Assim, recomendamos a escolha de um ou de outro método em função do objetivo do estudo. Sugerimos ainda que os dois métodos sejam empregados em um só estudo, como o experimento conduzido por Durigan et al. (2002a). Os autores utilizaram os métodos de QD para análise de riqueza e composição florística e o método de PA para determinação das densidades para superar as limitações inerentes de cada método. Por fim, sugerimos a condução de novos estudos em outras fitofisionomias do Cerrado e com distintas densidades e padrões de distribuição espacial dos indivíduos para confirmar ou refutar os resultados encontrados no presente estudo.

\section{Agradecimentos}

Ao Programa de Pós-graduação em Ecologia e Conservação da Universidade do Estado de Mato Grosso - UNEMAT pelo apoio financeiro; à Coordenação de Aperfeiçoamento de Pessoal de Nível Superior (CAPES) pela concessão de bolsa de estudo à F.C. Pereira, L. Maracahipes-Santos, S.L. Lima e K.S. Melo-Santos e de bolsa de Pós-Doutorado (PNPD) à H.A. Mews. 


\section{Referências}

APG III. An update of the angiosperm Phylogeny group classification for the orders and families of flowering plants: APG III. Botanical Journal of the Linnean Society, London, v. 161, p. 105-121, 2009.

AQUINO, A. G.; WALTER, B. M. T.; RIBEIRO, J. F. Dinâmica de populações de espécies lenhosas de cerrado, Balsas Maranhão. Árvore, Viçosa, v. 31, p. 793-803, 2007.

BRASIL, L. S.; GIEHL, N. F. S.; SANTOS, J. O.; SANTOS, A. O.; MARIMON, B. S.; MARIMON-JUNIOR, B. H. Efeito de borda sobre a camada de serapilheira em área de cerradão no leste de Mato Grosso. Biotemas, Florianópolis, v. 26, p. 37-47, 2013.

BUENO, B.; GALLETTI, V. S.; VICENTIN, W.; DAMASCENOJUNIOR, G. A.; POTT, A. Comparação de dois métodos fitossociológicos aplicados à mata ciliar do rio Miranda, Pantanal do Miranda, Corumbá, MS. In: FELFILI, J. M.; EISENLOHR, P. V.; MELO, M. M. R. F.; ANDRADE, L. A.; MEIRA-NETO, J. A. A. (Ed.). Fitossociologia no Brasil-Métodos e estudo de casos. Viçosa: Universidade Federal de Viçosa, 2011. p. 86-121.

COSTA, V. C.; OLIVERA, K. N.; NUNES, Y. R. F.; MENINO, G. C. O.; BRANDÃO, D. O.; ARAÚJO, L. S.; MIRANDA, W. O.; D'ANGELO-NETO, S. Florística e estrutura da comunidade arbórea de duas áreas de Cerrado sentido restrito no norte de Minas Gerais. Cerne, Lavras, v. 16, p. 267-281, 2010.

BRIDGEWATER, S.; RATTER, J. A.; FELIPE RIBEIRO, J. Biogeographic patterns, b-diversity and dominance in the cerrado biome of Brazil. Biodiversity and Conservation, New York, v. 13, n. 12, p. 2295-2317, 2004.

COTTAM, G.; CURTIS, J. T. The use of distance measures in phytosociological sampling. Ecology, New York, v. 37, p. 451-460, 1956.

DURIGAN, G.; NISHIKAWA, D. L. L.; ROCHA, E.; SILVEIRA, E. R.; PULITANO, F. M.; REGALADO, L. B.; CARVALHAES, M. A.; PARANAGUÁ, P. A.; RANIERI, V. E. L. Caracterização de dois estratos da vegetação em uma área de Cerrado no município de Brotas, SP, Brasil. Acta Botanica Brasilica, Feira de Santana, v. 16, p. 251-262, 2002a.

DURIGAN, G.; SANTOS, J.D.; GANDARA, F. B. Fitossociologia de dois fragmentos de floresta estacional semidecidual no pontal do Paranapanema, SP. Revista do Instituto Florestal, São Paulo, v. 14, p. 13-26, 2002b.

ENGEMAN, R. M.; SIGIHARA, R. T.; PANK, L. F.; DUSENBERRY, W. E. A comparison of plotless density estimators using monte carlo simulation. Ecology, New York, v. 75, p. 17691779, 1994.

FELFILI, J. M.; CARVALHO, F. A.; HAIDAR, R. F. Manual para monitoramento de parcelas permanentes nos biomas Cerrado e Pantanal. Brasília: Universidade de Brasília, 2005. 60 p.

FELFILI, J. M.; FELFILI, M. C.; NOGUEIRA, P. E.; ARMAS, J. F. S.; FARINAS, M. R.; NUNES, M.; SILVA-JÚNIOR, M. C.; REZENDE, A. V.; FAGG, C. W. Padrões fitogeográficos e sua relação com sistemas de terra no bioma Cerrado. In: SANO, S. M.; ALMEIDA, S. P.; RIBEIRO, J. F. (Ed.). Cerrado - Ecologia e flora. Brasília: Embrapa, 2008. p. 215-228.

FELFILI, J. M.; REZENDE, A. V.; SILVA-JÚNIOR, M. C. Biogeografia do Bioma Cerrado - Vegetação e solos da Chapada dos Veadeiros. Brasília: Universidade de Brasília, Finatec, 2007. $256 \mathrm{p}$.

FELFILI, J. M.; REZENDE, A. V.; SILVA-JÚNIOR, M. C; SILVA, M. A. Changes in the floristic composition of cerrado sensu stricto in Brazil over a nine-year period. Journal of Tropical Ecology, Winchelsea, v. 16, p. 579-590, 2000.

FELFILI, J. M.; SILVA-JÚNIOR, M. C. Biogeografia do bioma Cerrado - Estudo fitofisionômico da Chapada do Espigão Mestre do São Francisco. Brasília: Universidade de Brasília, 2001. 152 p.

FELFILI, J. M.; SILVA-JÚNIOR， M. C. Distribuição dos diâmetros numa faixa de cerrado na Fazenda Água Limpa (FAL) - DF. Acta Botanica Brasilica, Feira de Santana, v. 2, p. 85-104, 1988.

FELFILI, J. M.; FELFILI, J. M. Diversidade alfa e beta no cerrado sensu stricto da Chapada Pratinha, Brasil. Acta Botanica Brasilica. Belo Horizonte, v. 15, n. 2. P. 243-254, 2001.

FELFILI, J. M.; ROITMAN, I.; MEDEIROS, M. M.; SANCHEZ, M. Procedimentos e métodos de amostragem de vegetação. In: FELFILI, J. M.; EISENLOHR, P. V.; MELO, M. M. R. F.; ANDRADE, L. A.; MEIRA-NETO, J. A. A. (Ed.). Fitossociologia no Brasil - Métodos e estudo de casos. Viçosa: Universidade Federal de Viçosa, 2011. p. 86-121.

FONSECA, M. S.; SILVA-JÚNIOR, M. C. Fitossociologia e similaridade florística entre trechos de Cerrado sentido restrito em interflúvio e em vale no Jardim Botânico de Brasília, DF. Acta Botanica Brasilica, Feira de Santana, v. 18, p. 19-29, 2004.

GOMES, L.; LENZA, E.; MARACAHIPES, L.; MARIMON, B. S.; OLIVEIRA, E. A. Comparação florísticas e estruturais entre das comunidades lenhosas de cerrado típico e cerrado rupestre, Mato Grosso. Acta Botanica Brasilia, Feira de Santana, v. 25, p. 865-875, 2011.

GOMES, L.; MARACAHIPES, L.; MARIMON, B. S.; REIS, S. M. A; ELIAS, F.; SANTOS, L. M.; MARIMON JUNIOR, B. H.; LENZA, E. Post-fire recovery of savanna vegetation from rocky outcrops. Flora, Jena, v. 209, p. 201-208, 2014.

GORENSTEIN, M. R.; BATISTA, J. L. F.; DURIGAN, G. Influência do padrão espacial sobre a estimativa de densidade arbórea do método de quadrantes: um estudo por meio de simulação de Monte Carlo. Acta Botanica Brasilica, Feira de Santana, v. 21, p. 957-965, 2007.

GOTELLI, N. J.; COLWELL, R. K. Quantifying biodiversity: procedures and pitfalls in the measurement and comparison of species richness. Ecology Letters, Montpellier, v. 4, p.379-391, 2001.

HAMMER, Ø. PAlaeontological Statistics, version 3.0, Reference Manual. 2013. Disponível em: <http://folk.uio.no/ohammer/past/>. Acesso em: 3 nov. 2013.

LIBANO, A. M.; FELFILI, J. M. Mudanças temporais na composição florística e na diversidade de um cerrado sensu stricto do Brasil Central em um período de 18 anos (1985-2003). Acta Botanica Brasilica, Feira de Santana, v. 20, p. 927-936, 2006.

LISTA DE ESPÉCIES DA FLORA DO BRASIL. Jardim Botânico do Rio de Janeiro. 2014. Disponível em <http://floradobrasil.jbrj. gov.br/>. Acesso em: 26 maio 2014.

MAGURRAN, A. E. Ecological diversity and its measurement. London: Croom Helm, 1988. 179 p. 
MAGURRAN, A. E. Medindo a diversidade biológica. Curitiba: UFPR, 2011. $261 \mathrm{p}$.

MARIMON-JUNIOR, B. H.; HARIDASAN, M. Comparação da vegetação arbórea e características edáficas de um cerradão e um cerrado sensu stricto em áreas adjacentes sobre solo distrófico no leste de Mato Grosso, Brasil. Acta Botanica Brasilica, Feira de Santana, v. 19, p. 913-926, 2005.

MENDONÇA, R. C.; FELFILI, J. M.; WALTER, B. M. T.; SILVA-JÚNIOR, M. C.; REZENDE, A. B.; FILGUEIRAS, T. S.; NOGUEIRA, P. E.; FAGG, C. W. Flora vascular do Bioma Cerrado: checklist com 12.356 espécies. In: SANO, S. M.; ALMEIDA, S. P.; RIBEIRO, J. F. (Org.). Cerrado - Ecologia e flora. Brasília: Embrapa, 2008. p. 213-228.

MEWS, H. A.; PINTO, J. R. R.; EISENLOHR, P. V.; LENZA, E. Does size matter? Conservation implications of differing woody population sizes equivalent occurrence and diversity of species for threatened savanna habitats. Biodiversity and Conservation, New York, v. 23, p. 1119-1131, 2014.

MOREIRA, A. G. Effects of fire protection on savanna structure in Central Brazil. Journal of Biogeography, New York, v. 27, p. 1021-1029, 2000.

MORO, M. F.; MARTINS, F. R. Métodos de levantamento do componente arbóreo-arbustivo. In: FELFILI, J. M.; EISENLOHR, P. V.; MELO, M. M. R. F.; ANDRADE, L. A.; MEIRA-NETO, J. A. A. Fitossociologia no Brasil - Métodos e estudos de casos. Viçosa: Universidade Federal de Viçosa, 2011. p. 175-208.

MÜELlER-DOMBOIS, D.; ELlENBERG, H. (Ed.). Aims and methods of vegetation ecology. New York: John Wiley. 1974. 574 p. OKSANEN, J.; BLANCHET, F. G.; KINDT, R.; LENDRE, P.; MINCHIN, P. R.; O'HARA, R. B.; SIMPSON, G. L.; SOLYMOS, P.; STEVENS, M. H. H.; WAGNER, H. Vegan: Community Ecology Package. R package version 2.0-9. 2013. Disponível em: <http:/CRAN.R-project.org/package=vegan $>$. Acesso em: 22 maio 2014.

OLIVEIRA-FILHO, A. T.; RATTER, J. A. Vegetation physionomies and woody flora of the Cerrado biome. In: OLIVEIRA, P. S.; MARQUIS, R. J. (Ed.). The cerrados of Brazil. New York: Columbia University Press, 2002. p. 91-120.

PHILIP, M. S. Measuring trees and forests. Cambridge: Cambridge University Press. 1994. 324 p.

PINHEIRO E. S.; DURIGAN, G. Diferenças florísticas e estruturais entre fitofisionomias do cerrado em Assis, SP, Brasil. Árvore, Viçosa, v. 36, p. 181-193, 2012.

R CORE TEAM. R: a language and environment for statistical computing. Vienna: R Foundation for Statistical Computing, 2013. Disponível em $<$ http://www.R-project.org/>. Acesso em: 22 maio 2014.
RATTER, J. A.; BRIDGEWATER, S.; RIBEIRO, J. F. Analysis of the floristic composition of the Brazilian Cerrado vegetation III: comparison of the woody vegetation of 376 areas. Edinburgh Journal of Botany, Edinburgh, v. 60, p. 57-109, 2003.

RATTER, J. A.; GIFFORD, D. R. Observations on the vegetation of eastern Mato Grosso, Brasil. III. The woody vegetation and soils of the Morro de Fumaça, Torixoreu. Proceedings of the Royal Society, London, v. 235, p. 259-280, 1988.

RIBEIRO, J. F.; WALTER, B. M. T. As principais fitofisionomias do Bioma Cerrado. In: SANO, S. M.; ALMEIDA, S. P.; RIBEIRO, J. F. (Ed.). Cerrado: ecologia e flora. Brasília: Embrapa, 2008. p. 151-212.

SANTOS, T. R. R.; PINTO, J. R. R.; LENZA, E.; MEWS, H. A. The tree-shrub vegetation in rocky outcrop cerrado areas in Goias State, Brazil. Brazilian Journal of Botany, São Paulo, v. 35, p. 281-294, 2012.

SHEPHERD, G. J. Fitopac 2.1. Manual do usuário. Campinas: UNICAMP, 2010. Disponível em: <http://pedroeisenlohr.webnode. com.br/fitopac/>. Acesso em: 29 ago. 2014.

SILVA, J. F.; FARIÑAS, M. R.; FELFILI, J. M.; KLINK, C. A. Spatial heterogeneity, land use and conservation in the cerrado region of Brazil. Journal of Biogeography, New York, v. 33, p. 536-548, 2006.

SILVA, L. O.; COSTA, D. A.; ESPÍRITO SANTO FILHO, K.; FERREIRA, H. D. BRANDÃO, D. Levantamento florístico e fitossociológico em duas áreas de Cerrado sensu stricto no Parque Estadual da Serra de Caldas Novas, Goiás. Acta Botanica Brasilica, Feira de Santana, v. 16, p. 43-53, 2002.

SILVA-JÚNIOR, M. C. Fitossociologia e estrutura diamétrica da mata de Galeria do Taquara, na Reserva Ecológica do IBGE, DF. Árvore, Viçosa, v. 28, p. 419-428, 2004.

WALTER, B. M. T.; GUARINO, E. S. G. Comparação do método de parcelas com o "levantamento rápido" para amostragem da vegetação arbórea do Cerrado sentido restrito. Acta Botanica Brasilica, Feira de Santana, v. 20, p. 285-297, 2006.

WALTHER, B. A.; MOORE, J. L. The concepts of bias, precision and accuracy, and their use in testing the performance of species richness estimators, with a literature review of estimator performance. Ecography, Lund, v. 28, p. 815-829, 2005.

ZAR, J. H. Biostatistical analysis. 4. ed. New Jersey: Prentice Hall, 1999. 663 p. 